\title{
Acurácia do Teste de Clements Para Avaliação da Maturidade Pulmonar Fetal em Gestantes com Doença Hipertensiva Especifica da Gestação
}

\author{
Accuracy of Clements' Test for Evaluation of Fetal \\ Lung Maturation in Preeclamptic Patients
}

Melania Maria Ramos de Amorim, Aníbal Faúndes, Luiz Carlos Santos, Elvira Azevedo

\begin{abstract}
RESUM0
Objetivos: determinar sensibilidade, especificidade e valor preditivo positivo e negativo do teste de Clements para avaliação da maturação pulmonar fetal na DHEG.

Métodos: foi realizado um estudo prospectivo para validação de técnica diagnóstica, envolvendo 163 gestantes (com idade gestacional entre 28-34 semanas) internadas no IMIP com diagnóstico de DHEG (leve, grave, pura ou superposta) e indicação de pesquisa de maturidade pulmonar fetal. O diagnóstico de DHEG e suas formas clínicas foi realizado de acordo com os parâmetros propostos pelo "National High Blood Pressure Working Group", 1990. O teste de Clements foi realizado em três tubos, considerando-se para análise os resultados positivos, intermediários e negativos (em relação à presença ou não de maturação pulmonar fetal). Os parâmetros de acurácia já descritos foram calculados levando em consideração a real incidência de doença da membrana hialina (maturidade positiva = ausência de doença) ao nascimento. A análise dos dados foi realizada em Epi-Info 6.04, utilizando-se o teste $\chi^{2}$ de associação e considerando-se um nível de significância de 5\%. Resultados: para determinação da acurácia do método, foram considerados os resultados intermediários como positivos ou negativos. Quando estes foram considerados positivos, determinou-se uma sensibilidade de $87,9 \%$ e uma especificidade de 74,5\%, valor preditivo positivo e negativo de respectivamente $89,4 \%$ e $71,4 \%$, com uma acurácia de $84 \%$. Em se analisando como negativos esses resultados intermediários, a sensibilidade caiu para 62\%, ao passo que a especificidade elevou-se para 89,4\%, com um valor preditivo positivo de 93,5\% e valor preditivo negativo de 51,2\% (acurácia em torno de 70\%). Os resultados falsopositivos foram raros e em geral associados à hipoxia neonatal: somente 5 (6.5\%) de 77 recém-nascidos com Clements positivo apresentaram DMH após o nascimento. No entanto, os resultados falso-negativos foram freqüentes, em torno de $40 \%$ para os resultados negativos/intermediários.
\end{abstract}

Instituto Materno - Infantil de Pernambuco (IMIP) -

Centro de Atenção à Mulher (CAM)

Correspondência:

Melania Maria Ramos de Amorim

IMIP - Rua dos Coelhos, 300 - Ilha do Leite

50070-530 - Recife-PE

e-mail: andfpc@br.homeshopping.com.br 
Conclusões: o teste de Clements representa um bom teste para investigação maturação pulmonar fetal em casos de DHEG, sendo raros os resultados falso-positivos. No entanto, em virtude da especificidade baixa, com elevada freqüência de resultados falso-negativos, seus resultados devem ser analisados com cautela, complementando-se a pesquisa de maturidade com outros métodos (sobretudo em casos graves, quando a maturidade pulmonar presente irá determinar a indicação de interrupção da gestação).

PALAVRAS-CHAVE: Teste de Clements. Maturidade Pulmonar Fetal. DHEG. Pré-eclâmpsia; Doença da Membrana Hialina.

\section{Introdução}

A prematuridade constitui, ainda em nossos dias, uma das complicações mais freqüentes da Doença Hipertensiva Específica da Gestação $(\mathrm{DHEG})^{4,13}$, decorrente quer de um trabalho de parto espontâneo, em razão quer da contratilidade uterina aumentada quer, comumente, da conduta obstétrica de interrupção da gravidez, quando o quadro clínico se agrava e há comprometimento das condições maternas ou fetais. Determinada pela presença do vilo corial no organismo materno ${ }^{4,17}$, de fato, a única "cura" possível para a doença é o parto, e o parto prematuro terapêutico é indicado, amiúde, em idade gestacional precoce, quando soem apresentar-se as formas graves.

Dessa forma, expressivo percentual das complicações neonatais relacionadas à DHEG é provocado pela prematuridade ${ }^{6}$ e a decisão pelo parto, embora geralmente baseada na gravidade da doença, requer, desde que não exista risco aumentado para a mãe, a avaliação de maturidade pulmonar do concepto ${ }^{19}$.

A Doença da Membrana Hialina (DMH), ou Síndrome de Angústia Respiratória do RecémNascido (SAR) é de longe o problema clínico mais comumente encontrado entre os recém-nascidos ${ }^{9,16}$. A incidência varia em função da idade gestacional: entre 28-30 semanas é de aproximadamente 70\%, diminuindo então progressivamente, de forma que é excepcional sua ocorrência a partir de 37 semanas $^{5}$. No IMIP, sua incidência em prematuros de mães com DHEG entre 28-34 semanas é de $42 \%{ }^{2}$.

Sua importância como causa de mortalidade neonatal persiste, atualmente, apesar do real progresso obtido em termos de prevenção ou redução de sua gravidade, promovido em parte pelos avanços obstétricos na prevenção do parto prematuro e na aceleração farmacológica da maturidade pulmonar e, por outro lado, pelo emprego da terapia pós-natal com surfactante exógeno ${ }^{10}$.

Em decorrência dessa preocupação com a prematuridade, deve, portanto, o obstetra avaliar os riscos e benefícios de cada caso e realizar uma boa avaliação da maturidade fetal. Essa avaliação pode ser feita por meio de métodos clínicos e ecográficos (baseados na determinação da idade gestacional), bem como pelo estudo do líquido amniótico. Nesse, são analisadas as características físicas, a bioquímica e a citologia. Podem ser realizados o TAP teste ${ }^{22}$, a espectrofotometria, a dosagem de fosfatidilglicerol ${ }^{1,8}$, a relação lecitina/ esfingomielina ${ }^{7}$ e o teste de Clements.

Em 1972, Clements et $a .^{3}$ propuseram um teste rápido, simples e seguro para avaliar a maturidade fetal, que se baseia na capacidade que o surfactante pulmonar tem de formar bolhas estáveis quando em contato com o etanol. O tubo é considerado positivo quando há bolhas formando um anel completo, intermediário quando há bolhas, mas que não completam o anel e negativo quando não há bolhas. O teste, por sua vez, é positivo quando os três tubos apresentam bolhas completas, negativo quando não há formação de bolhas em nenhum dos tubos e intermediário nas demais condições ${ }^{3,11}$.

No que diz respeito à acurácia do teste de Clements, ele praticamente não apresenta resultados falso-positivos, variando de $1-3 \%^{20}$; entretanto, os falso-negativos são mais freqüentes variando de $8 \%$ a $10 \%$ para alguns autores ${ }^{11}$ e de $10 \%$ a $40 \%$ para outros. Cunningham et al. ${ }^{4}$ acreditam que é necessária uma relação L/E de 4,0 ou superior para produzir um teste positivo. Nos casos de contaminação com sangue pode haver resultados falso-positivos, problema esse que se resolve com a centrifugação do líquido amniótico.

O presente estudo foi realizado com o objetivo de determinar sensibilidade, especificidade, valor preditivo positivo e negativo do teste de Clements para avaliação da maturação pulmonar fetal em 
gestações complicadas pela Doença Hipertensiva Específica da Gestação.

\section{Pacientes e Métodos}

Foi realizado um estudo prospectivo para validação de técnica diagnóstica, envolvendo 163 gestantes (com idade gestacional entre 28 - 34 semanas) internadas no IMIP com diagnóstico de DHEG (leve, grave, pura ou superposta) e indicação de pesquisa de maturidade pulmonar fetal.

Os critérios de inclusão foram: idade gestacional maior ou igual a 28 e menor que 35 semanas (ou seja, até 34 semanas e 6 dias), confirmada pela data da última menstruação (DUM) e/ou ultra-sonografia, compativel com a avaliação clínica do recém-nascido (método de Capurro); diagnóstico materno confirmado de DHEG (avaliação clínica e laboratorial); concepto vivo; gestação única.

Os critérios de exclusão foram eclâmpsia, doenças maternas associadas (diabetes, colagenoses, cardiopatias, asma), amniorrexe prematura, presença de mecônio ou sangue no líquido amniótico por ocasião da amniocentese, líquido amniótico meconizado diagnosticado durante o trabalho de parto com ou sem Síndrome de Aspiração Meconial, morte neonatal ocorrida antes de 24 horas de vida, tempo decorrido entre a amniocentese e o parto maior que 1 semana, terapia prévia com corticosteróides durante a gravidez (betametasona, hidrocortisona, dexametasona); placenta prévia - ou qualquer episódio hemorrágico no $2^{\circ}$ ou $3^{\circ}$ trimestre; Doença Hemolítica Perinatal e recém-nascidos com malformações congênitas.

As variáveis pesquisadas foram o resultado do teste de Clements (independente) e ocorrência de Doença da Membrana Hialina (dependente).

\section{Definição de Termos, Procedimentos e Variáveis}

DHEG - hipertensão (PAS $\geq 140$ e/ou $\mathrm{PAD} \geq 90 \mathrm{mmHg}$ ), proteinúria (1+ pelo labstix em 2 ocasiões ou $2+$ ou mais em dosagem isolada ou ainda $\geq 300 \mathrm{mg} / 1 / 24$ horas), hiperuricemia (ácido úrico $\geq 4.5 \mathrm{mg} \%$ )

DHEG grave - diagnóstico de qualquer um dos seguintes sinais e sintomas em pacientes com pressão arterial $\geq 160 / 110 \mathrm{mmHg}$, proteinúria $\geq 2 \mathrm{~g} / 1 / 24$ horas ou labstix de $3+$ ou mais, oligúria / oligoanúria ( $\downarrow 25 \mathrm{ml} /$ hora), creatinina sérica $>1,2 \mathrm{mg} \%$, sinais e sintomas de eclâmpsia iminente, achados clínicos ou laboratoriais de HELLP sindrome (plaquetas abaixo de $100.000 / \mathrm{mm}^{3}$, transaminases $\uparrow 70 \mathrm{UF}$, bilirrubinas > 1,2mg\%), edema agudo de pulmão ou cianose, achados fundoscópicos - hemorragias, exsudatos retinianos e papiledema ${ }^{15}$.

DHEG superposta - associação dos parâmetros clínicos ou laboratoriais da DHEG em pacientes com hipertensão arterial crônica.

Doença da Membrana Hialina (Síndrome de Angústia Respiratória do Recém-Nascido) - o diagnóstico foi estabelecido a partir dos critérios definidos pelo CLAP $^{21}$ : sinais de insuficiência respiratória nas primeiras horas de vida (dispnéia, taquipnéia $>60 /$ minuto, retração esternal, tiragem intercostal, cianose central e gemido expiratório) com piora progressiva e surgimento de crises de apnéia, atingindo o pico entre 48-72 horas; achados radiológicos - padrão reticulogranular difuso, com ou sem aerobroncograma; necessidade de assistência respiratória com 24 horas de vida.

Para a avaliação pré-natal da maturidade pulmonar fetal foram considerados os resultados do teste de Clements, realizado no líquido amniótico obtido através de amniocentese a partir da $30^{\mathrm{a}}$ semana de gestação.

Para o teste de Clements são utilizados três tubos, de diâmetro idêntico (8-14 mm), contendo diluições sucessivas do líquido amniótico: $1 \mathrm{ml}$, $0.75 \mathrm{ml}$ e $0.5 \mathrm{ml}$, completados para $1 \mathrm{ml}$ pela adição de soro fisiológico. Acrescenta-se $1 \mathrm{ml}$ de etanol a 95\%, agitando-se os tubos por 15 segundos e realizando-se a leitura após 15 minutos ${ }^{11}$. Os tubos são "lidos" interpretando-se como resultado positivo no tubo a presença de bolhas em toda a sua volta, negativo quando não se formam bolhas e intermediário quando as bolhas não ocorrem em toda a circunferência do tubo ${ }^{3,11}$.

Considerou-se imaturidade pulmonar (teste negativo) - imaturidade pulmonar quando havia resultado negativo no segundo e no terceiro tubo e negativo ou intermediário no primeiro tubo.

Considerou-se (teste positivo) - maturidade pulmonar quando o resultado foi positivo nos 2 primeiros tubos e positivo ou intermediário no terceiro e teste intermediário nas condições restantes.

A análise estatística foi realizada com o programa estatístico Epi-Info 6.04b, de domínio público, utilizando-se médias e seus desvios-padrão e teste "t" de Student para as variáveis quantitativas, e os testes $\chi^{2}$ de associação e exato de Fisher para as variáveis qualitativas, considerando-se um nível de significância de 5\% .

Os resultados do teste de Clements foram analisados de duas formas: a primeira considerando-se como negativos os resultados 
intermediários e a segunda incluindo esses últimos como positivos.

Dicotomizada a variável de análise, foram construídas tabelas de contingência para determinar a associação dos resultados do teste de Clements com a presença ou não de Doença da Membrana Hialina, utilizando-se o teste do $\chi^{2}$ de Pearson, para um nível de significância de $5 \%$.

Para a análise da acurácia do método, foram testados os parâmetros Sensibilidade, Especificidade, Valor Preditivo Positivo e Negativo. Considerou-se para determinação desses parâmetros que uma peculiaridade do teste de Clements é a positividade relacionada à presença de maturidade (ou seja, ausência de doença).
TESTE DE CLEMENTS

\section{Positivo para DMH}

(Resultado Negativo)

Negativo para DMH

(Resultado Positivo)

$\mathrm{c}$

Sensibilidade $=\mathrm{a}: \mathrm{a}+\mathrm{c}$

Especificidade $=\mathrm{d}: \mathrm{b}+\mathrm{d}$

Valor preditivo positivo $=\mathrm{a}: \mathrm{a}+\mathrm{b}$

Valor preditivo negativo $=\mathrm{d}: \mathrm{c}+\mathrm{d}$

Os parâmetros de acurácia já descritos foram calculados levando em consideração a real incidência de Doença da Membrana Hialina (maturidade positiva $=$ ausência de doença) ao nascimento.

\section{Resultados}

As diversas formas clínicas da DHEG entre as pacientes envolvidas no presente estudo estão expostas na Tabela 1. Quase 50\% dessas tinham DHEG grave "pura" e as restantes DHEG leve ou superposta (28,8\% e $23,3 \%$, respectivamente).

Na Tabela 2 são apresentadas características maternas diversas, como a idade (média de 24,4 anos), o número de gestações (média de 2,04) e a idade gestacional na amniocentese e no parto (ambas com média de 33 semanas).
Tabela 1 - Distribuição das pacientes de acordo com a forma clínica da DHEG

\begin{tabular}{lcc} 
Forma Clínica & $\mathbf{n}^{\mathbf{0}}$ & $\mathbf{\%}$ \\
\hline DHEG leve & 47 & 28,8 \\
DHEG grave & 78 & 47,9 \\
DHEG superposta & 38 & 23,3 \\
Total & 163 & 100,0
\end{tabular}

Tabela 2 - Algumas características maternas (média e DP)

Característica

Média e Intervalo de Confiança

Idade (anos)

$24,4 \pm 0,9$

Número de gestações

$2,04 \pm 0,25$

Idade gestacional na amniocentese

$33,0 \pm 1,8$

Idade gestacional no parto

$33,0 \pm 0,2$
Em relação à evolução da gestação (Tabela 3), o intervalo médio entre amniocentese e parto foi de 1,22 dias. Quase 50\% das gestantes cursaram com dopplerfluxometria umbilical alterada (aumento da resistência, diástole zero ou reversa) ou centralização do fluxo (relação artéria cerebral média/artéria umbilical < 1,0). As principais indicações de interrupção da gestação foram sofrimento fetal $(44,2 \%)$ e maturidade pulmonar fetal $(17,2 \%)$. A interrupção foi realizada na maioria dos casos por via alta (cesariana em $63,2 \%$ das pacientes).

Tabela 3 - Evolução da gestação

\section{Evolução da Gestação}

Sofrimento Fetal (doppler alterado)

$48 / 97(49,5 \%)$

Intervalo amniocentese-parto (média em dias)

$1,22 \pm 0,24$

Indicação de interrupção da gestação

- Sofrimento Fetal

$72(44,2 \%)$

- Maturidade Pulmonar Fetal

$28(17,2 \%)$

- Indicações maternas

$63(38,7 \%)$

Via de parto

- Parto transpélvico

$60(36,8 \%)$

- Cesariana

$103(63,2 \%)$

Resultados positivos do teste de Clements foram mais freqüentes $(47,2 \%)$, seguindo-se os negativos $(30,1 \%)$ e intermediários $(22,7 \%)$, conforme se observa na Tabela 4. Para a análise subseqüente, os resultados intermediários foram considerados como negativos. 
Tabela 4 - Distribuição de acordo com os resultados do Teste de Clements

\begin{tabular}{lcc}
\hline Resultado do Clements & $\mathbf{N .}^{\mathbf{0}}$ & $\boldsymbol{\%}$ \\
\hline Negativo & 49 & 30,1 \\
Intermediário & 37 & 22,7 \\
Positivo & 77 & 47,2 \\
Total & 163 & 100,0 \\
\hline
\end{tabular}

Na Tabela 5 são analisados alguns parâmetros relacionados à evolução da gestação de acordo com os resultados do teste de Clements, observando-se uma idade gestacional ligeiramente maior no grupo que apresentou resultados positivos, tanto na época da amniocentese como do parto (aproximadamente 33,5 versus 32,7 semanas). Sofrimento fetal anteparto, diagnosticado como alteração da dopplerfluxometria fetal, foi mais freqüente no grupo com resultados negativos $(61,2 \%$ contra $39,6 \%, p=0,03)$. O percentual de cesarianas também apresentou diferença estatisticamente significativa entre os dois grupos $(73,9 \%$ quando o resultado foi positivo e $51,9 \%$ quando o resultado foi negativo).

Tabela 5 - Resultados gestacionais de acordo com o resultado do Teste de Clements

\begin{tabular}{lccc}
\hline Parâmetro & $\begin{array}{c}\text { Clements } \\
\text { Positivo }\end{array}$ & $\begin{array}{c}\text { Clements } \\
\text { Negativo * }\end{array}$ & p \\
\hline $\begin{array}{l}\text { Idade gestacional média } \\
\text { (amniocentese) }\end{array}$ & $33,5 \pm 0,3$ & $32,7 \pm 0,5$ & NS ** \\
$\begin{array}{l}\text { Sofrimento Fetal Anteparto } \\
\quad \text { (Doppler alterado) }\end{array}$ & $19 / 48(39,6 \%)$ & $30 / 49(61,2 \%)$ & $<0,05$ \\
$\begin{array}{l}\text { \% cesarianas } \\
\text { Idade gestacional - Capurro } \\
\text { (média em semanas) }\end{array}$ & $33,4 \pm 0,2$ & $32,7 \pm 0,3$ & $<0,001$ \\
& & &
\end{tabular}

* Resultados intermediários incluídos como negativos

** Não significativo

Os principais resultados perinatais são apresentados na Tabela 6 . O peso ao nascer foi em média quase 200 gramas maior entre os recémnascidos com resultados positivos do teste de Clements $(p=0,002)$. O percentual de recémnascidos de muito baixo peso (abaixo de 1500 gramas) foi significativamente maior nos recémnascidos com resultados negativos $(p=0,0001)$. Escores de Apgar tanto no primeiro como no quinto minuto foram mais baixos nesse último grupo. $\mathrm{O}$ percentual de Apgar no quinto minuto abaixo de 7 foi de $3,9 \%$ para os casos com Clements positivo e $12,8 \%$ para os casos com Clements negativo $(p=0,04)$.

Em relação à incidência de DMH (V. Tabela 6), essa foi quase oito vezes maior no grupo com Clements negativo: $48,8 \%$ versus $6,5 \%$ no grupo com Clements positivo ( $p=0,0000001)$. A incidência global de $\mathrm{DMH}$ (no total de 163 neonatos) foi de $25,8 \%$.

Tabela 6 - Características perinatais de acordo com o resultado do clements

\begin{tabular}{lrrr}
\hline Parâmetro & $\begin{array}{c}\text { Clements } \\
\text { Positivo }\end{array}$ & $\begin{array}{c}\text { Clements } \\
\text { Negativo * }\end{array}$ & p \\
\hline Peso ao nascer & & & \\
(média em gramas) & $1718 \pm 80,1$ & $1544 \pm 77,6<0,01$ \\
\% abaixo de 1500 gramas & $17 / 77(22,1 \%)$ & $41 / 86(47,7 \%)$ & \\
\% Apgar de $1^{\circ}$ minuto $<7$ & $9 / 77(11,7 \%)$ & $34 / 86(39,5 \%)$ & $<0,01$ \\
\% Apgar de $5^{\circ}$ minuto $<7$ & $3 / 77(3,9 \%)$ & $11 / 86(12,8 \%)$ & $<0,05$ \\
Incidência de DMH & $5 / 77(6,5 \%)$ & $42 / 86(48,8 \%)$ & $<0,001$ \\
\hline
\end{tabular}

* Os resultados intermediários foram incluídos como negativos

Analisando-se os resultados intermediários do teste de Clements como negativos (V. Tabela 7), observou-se uma boa especificidade (Clements positivo como preditor da ausência de doença). Os testes falso-positivos foram infreqüentes (pouco mais de $10 \%$ ), caracterizando-se um elevado valor preditivo positivo. Quando foram analisados individualmente os casos de falso-positivos, constatou-se que, em sua maioria, associavam-se à hipoxia intraparto. A acurácia ficou em torno de $70 \%$, refletindo a sensibilidade pouco acima de $60 \%$.

Quando os resultados intermediários foram incluídos na análise como positivos (Tabela 8), a especificidade caiu em torno de 15 pontos percentuais, mas a sensibilidade subiu mais de 20 pontos. Conseqüentemente, ocorreu piora do valor preditivo positivo e melhora do valor preditivo negativo.

Tabela 7 - Acurácia do Teste de Clements considerando-se como negativos os resultados intermediários

\begin{tabular}{ll}
\multicolumn{1}{c}{ Parâmetro } & \\
\hline Sensibilidade & $62,0 \%$ \\
Especificidade & $89,4 \%$ \\
Taxa de falso-positivos & $10,6 \%$ \\
Taxa de falso-negativos & $38,0 \%$ \\
Valor preditivo ositivo & $93,5 \%$ \\
Valor preditivo negativo & $51,2 \%$ \\
Acurácia & $69,9 \%$
\end{tabular}


Tabela 8 - Acurácia do Teste de Clements considerando-se como positivos os resultados intermediários

\begin{tabular}{ll}
\multicolumn{1}{c}{ Parâmetro } & \\
\hline Sensibilidade & $87,9 \%$ \\
Especificidade & $74,5 \%$ \\
Taxa de falso-positivos & $25,5 \%$ \\
Taxa de falso-negativos & $12,1 \%$ \\
Valor preditivo positivo & $89,4 \%$ \\
Valor preditivo negativo & $71,4 \%$ \\
Acurácia & $84,0 \%$
\end{tabular}

\section{Discussão}

Os resultados do presente estudo evidenciam uma acurácia do teste de Clements variando de 70 - 85\%, de acordo com a interpretação dos testes intermediários (analisados alternadamente como positivos ou negativos). O método apresenta uma elevada especificidade, sendo raros os testes falsopositivos, porém a sensibilidade para maturidade pulmonar fetal é relativamente baixa, com resultados falso-negativos entre $12-38 \%$. Esses resultados corroboram aqueles descritos pela literatura de acordo com diversos autores ${ }^{4,12}$ resultados da relação L/E tão elevados como 4-6 seriam necessários para a obtenção de um teste positivo.

No entanto, o valor preditivo de um teste positivo para a presença de maturidade pulmonar fetal (isto é, para a ausência de $\mathrm{DMH}$ ) é bastante elevado, aproximando-se de $95 \%$, o que confere uma elevada confiabilidade ao teste quando as bolhas se formam nos três tubos. Assim, muitos autores têm proposto que o teste de Clements seja utilizado para triagem, acreditando-se nos resultados positivos ${ }^{19}$ e submetendo as pacientes com resultados negativos a novos testes, mais sensíveis, como a relação $\mathrm{L} / \mathrm{E}$, o fosfatidilglicerol ou outros.

Vale ressaltar que os testes falsos positivos encontrados nesse estudo, correspondendo a casos de Doença da Membrana Hialina em recémnascidos de mães com resultados positivos do Clements, ocorreram todos em recém-nascidos hipoxiados, com baixos escores de Apgar no quinto minuto. A hipoxia perinatal tem sido descrita por vários autore $\mathrm{s}^{5,9}$ como fator de risco para DMH e é bastante frequente na vigência da DHEG. Assim é possivel que estoques de surfactante suficientes para positivar o teste de Clements tenham diminuído em decorrência da asfixia. Em outro estudo recentemente realizado no IMIP, Carvalho ${ }^{2}$ encontrou uma especificidade mais elevada $(97,8 \%)$ para o teste de Clements em gestantes normotensas, com uma taxa de falsos positivos de apenas $2,2 \%$.

Esses resultados assumem fundamental importância na conduta obstétrica em pacientes com DHEG: o momento "ideal" para a interrupção da gestação nesses casos constitui muitas vezes um dilema para o obstetra, pois o parto constitui de fato a única "cura" até hoje conhecida para o processo de vasoespasmo desencadeado pela presença do vilo corial no organismo. Sua antecipação, se oportuna, é, não raras vezes, decisiva para o bom prognóstico materno, e ainda pode representar a libertação do concepto de um meio intra-uterino hostil, dominado pela hipoxia resultante da insuficiência placentária. A questão crucial, no entanto, é como atuar para que essa antecipação não resulte no nascimento de prematuros que venham a falecer em decorrência da imaturidade pulmonar, desenvolvendo Doença da Membrana Hialina.

A antiga impressão clínica de uma maturação pulmonar acelerada na DHEG não tem sido confirmada por estudos utilizando métodos mais $\operatorname{acurados}^{2,19}$ que apontam para uma incidência de DMH semelhante entre conceptos de mães com DHEG e normotensas. À luz das evidências atuais, a decisão obstétrica de interrupção da gestação não se pode firmar na presunção de uma "possível" maturidade pulmonar em conceptos por vezes extremamente prematuros.

Se a deterioração das condições maternas impõe por vezes essa decisão, não são raras as ocasiões em que, assegurado o bem-estar materno, o concepto pode ser beneficiado pela permanência intra-uterina. Essa constatação tem levado ao redimensionamento da importância dos testes de avaliação anteparto da maturidade fetal. Schiff et al. ${ }^{19}$ apontam a necessidade da avaliação da maturidade pelos testes bioquímicos, ao invés da mera presunção.

Em serviços e regiões onde não há disponibilidade de realização desses testes, como no IMIP e em diversos Estados brasileiros, impõese, portanto, a análise dos parâmetros de acurácia do teste de Clements, visando nortear a conduta obstétrica. Acreditamos que o presente estudo traz subsídios importantes nesse aspecto: diante de resultados positivos deve-se admitir a presença de maturidade pulmonar fetal, estando portanto indicada a interrupção terapêutica da gestação. Persiste todavia o questionamento acerca da conduta obstétrica quando são encontrados resultados negativos e o teste de Clements é o único método disponivel para avaliação da maturidade pulmonar fetal.

A utilização do teste TAP, preconizada por 
Socol et al. ${ }^{22}$, tem sido adotada em alguns centros ${ }^{12}$ pelo seu baixo custo, fácil execução e elevado valor preditivo para maturidade pulmonar fetal. No entanto, os falso-negativos são ainda freqüentes ${ }^{14}$.

A tendência atual no IMIP é de realizar o teste TAP em associação com o teste de Clements e a interrupção da gestação é indicada sempre que os resultados indicam a presença de maturidade pulmonar fetal. Diante de um resultado negativo, há que se considerar a gravidade da doença, a idade gestacional, o peso estimado e as condições de vitalidade fetal. O sofrimento fetal constitui indicação de parto prematuro terapêutico independente dos resultados do teste de Clements. Em pacientes com DHEG grave, a conduta conservadora só é indicada nos conceptos com maturidade ausente abaixo de 35 semanas. A partir de 35 semanas, preconiza-se a interrupção da gravide $z^{18}$.

Casos com idade gestacional entre 30-34 semanas com resultados negativos do Clements ou abaixo de 30 semanas são submetidos à corticoterapia com betametasona (12 mg IM semanalmente). Como os resultados do teste de Clements não são afetados pelo uso de corticosteróides, a interrupção é realizada a partir de 34 semanas, sem necessidade de repetição da amniocentese ${ }^{18}$.

Essa conduta parece justificada à luz das evidências apontadas pelo presente estudo: embora outros critérios devam ser considerados, como a estimativa de peso fetal e o limite de viabilidade fetal (que depende sobretudo das condições da Unidade de Neonatologia), a obtenção de um resultado positivo do teste de Clements constitui um indicador seguro de maturidade pulmonar fetal.

Concluindo, podemos afirmar que a sensibilidade do teste de Clements foi de $62 \%$ quando os resultados intermediários foram considerados negativos e $88 \%$ quando esses resultados foram considerados positivos. Da mesma forma, a especificidade foi de $89,4 \%$ e $74,5 \%$, respectivamente, e, portanto, esse teste representa um bom método de triagem da maturação pulmonar fetal em casos de DHEG, sendo raros os resultados falsos-positivos.

No entanto, em razão da elevada freqüência de resultados falso-negativos, seus resultados devem ser analisados com cautela, complementando-se a pesquisa de maturidade com outros métodos (sobretudo em casos graves, quando a maturidade pulmonar presente irá determinar a indicação de interrupção da gestação).

\section{SUMMARY}

Objectives: To determine sensitivity, specificity, positive and negative predictive values of the shake test (Clements) for evaluation offetal lung maturation in preeclamptic patients.

Methods: A prospective study for validation of a diagnostic method was conducted enrolling 163 preeclamptic patients (gestational age between 28-34 weeks) admitted at CAM-IMIP with indication for fetal maturity testing. Preeclampsia diagnosis and classification followed criteria of the National High Blood Pressure Working Group, 1990. Clements' test was performed in three tubes and positive, negative or intermediate results were considered for analysis (related to presence or absence of fetal lung maturity). Accuracy parameters were calculated considering actual incidence of hyaline membrane disease (positive maturity = absent disease) after birth. Hyaline membrane disease was defined by criteria of CLAP, 1978. Statistical analysis was performed using $\chi^{2}$ test (Epi-Info 6.04b) with a 5\% significance level.

Results: Intermediate results were considered alternately as positive or negative for analysis. When considered positive, sensitivity was $87.9 \%$ and specificity $74.5 \%$ with positive and negative predictive values of $8.9 .4 \%$ and $71.4 \%$ respectively efficiency was $84 \%$. When intermediate results were evaluated as negative, sensitivity decreased to $62 \%$ and specificity raised to $89.4 \%$ and positive and negative predictive values were $93.5 \%$ and $51.2 \%$ respectively (efficiency $=70 \%$ ). False-positive results were rare and usually related to neonatal hypoxia: only $5(6.5 \%)$ of 77 neonates with previous positive Clements had hyaline membrane disease. Nevertheless, false negatives were frequent: almost $40 \%$ for negative/intermediate results.

Conclusions: Despite its limitations, Clements' test remains a good method for investigation of fetal lung maturation in preeclamptic patients since false positive results are unusual. However sensitivity is low and results have be cautiously analyzed because of elevated rate of false negative results. A good policy is to complement fetal maturity investigation with other tests if a negative result is determined, specially in severe cases when confirmed maturity represents indication for interruption of pregnancy.

KEY WORDS: Clements' test. Fetal lung maturation. Preeclampsia. Hyaline membrane disease.

\section{Referências}

1.Bustos R, Kulovich MV, Gluck L, Gabbe SG, Evertson L, Vargas C, et al. - Significance of phosphatidylglycerol in amniotic fluid in complicated pregnancies. Am J Obstet Gynecol 1979; 133: 899 - 903.

2.Carvalho MMRA. Avaliação da maturidade pulmonar na Doença Hipertensiva Específica da Gestação (DHEG). Tese (Mestrado) Recife: Instituto Materno-Infantil de Pernambuco; 1995. 
3.Clements JA, Platzker ACG, Tierney DF, Hobel CJ, Creasy RK, Margolis AJ, et al. Assessment of the risk of respiratory-distress syndrome by a rapid test for surfactant in amniotic fluid. N Engl $\mathbf{J}$ Med 1972; 286: 1077-82.

4.Cunningham FG, Mac Donald PC. Hypertensive disorders in pregnancy. In: Cunningham FG, William SJ. Whittidge. Williams Obstetrics, $20^{\text {th }}$ ed. Norwalk: Appleton \& Lange; 1997.

5.Fanaroff AA, Martin RJ, Miller MJ. Identification and management of high-risk problems in the neonate. In: Creasy RK, Resnik R. Maternal-Fetal Medicine. 3rd ed. Philadelphia: W. B. Saunders; 1994. p.1141-4.

6.Friedman SA, Schiff E, Kaoli Sibai BM. Neonatal outcome after preterm delivery for preeclampsia. Am J Obstet Gynecol 1995; 172: 1785-92.

7.Gluck L, Kulovich MV, Borer RC Jr, Keidel WN. The interpretation and significance of the lecithin-sphingomyelin ratio in amniotic fluid. Am J Obstet Gynecol 1973; 115: 539-46.

8.Hallman M, Kulovich MV, Kirkpatrick E, Sugarman RC, Gluck L. Phosphatidylinositol and phosphatidylglicerol in amniotic fluid: indices of lung maturity. Am J Obstet Gynecol 1976; 125:613-7.

9.Hjalmarson O. Epidemiology and classification of acute, neonatal respiratory disorders. Acta Paediatr Scand 1981; 70: 773-83.

10.Jobe AH. Lung maturational agents and surfactant treatments: are they complementary in preterm infants? J Perinatalol 1989; 9: 14-8.

11.Kondo MM. Provas de maturidade fetal. In: Cha SC, Zugaib M editores. Medicina Fetal. São Paulo: Atheneu 1993. p.119-25.

12.Maberry MC. Methods to diagnose fetal lung matu- rity. Semin Perinatol 1993; 17:241-6.

13.Main DM, Gabbe SG, Richardson D, Strong S. Can preterm deliveries be prevented? Am J Obstet Gynecol 1985; 151: 892-8.

14. Martins-Costa SH, Ramos JGL. Avaliação da maturidade pulmonar fetal. In: Freitas F, Martins-Costa SH, editores. Rotinas em Obstetrícia. Porto Alegre: Artes Médicas; 1997. p. 98-103.

15.National High Blood Pressure Education Program Working Group Report on High Blood Pressure in Pregnancy (Consensus Report). Am J Obstet Gynecol 1990; 163: 1691-712.

16.Perelman RH, Farrel PM. Analysis of causes of neonatal death in the U.S. with specific emphasis on fatal hyaline membrane disease. Pediatrics 1982; 70: 570-5.

17.Pritchard JA. Management of severe preeclampsia and eclampsia. Semin Perinatol 1978; 2: 83-97.

18.Santos LC, Porto AMF, Carvalho MMRA, Guimarães V. IMIP - Obstetrícia: Diagnóstico e Tratamento. Rio de Janeiro: Medsi; 1998.

19.Schiff E, Friedman SA, Mercer BM, Sibai BM. Fetal lung maturity is not accelerated in preeclamptic pregnancies. Am J Obstet Gynecol 1993; 169: 1096-101.

20.Schlueter MA, Phibbs RH, Creasy RK, Clements JÁ, Tooley WH. Antenatal prediction of graduated risk of hyaline membrane disease by amniotic fluid foam test for surfactant. Am J Obstet Gynecol 1979; 134: 761-5.

21.Schwarcz R, Capurro H. Manejo Perinatal de la Premature. Centro Latinoamericano de Perinatologia e Desarrollo Humano; 1978. Pub. Cient. 755.

22.Socol ML, Sing E, Depp R. The Tap test: a rapid indicator of fetal pulmonary maturity. Am J Obstet Gynecol 1984;148: 445-50. 